\title{
Wedelolactone inhibits breast cancer-induced osteoclastogenesis by decreasing Akt/mTOR signaling
}

\author{
CHIA-JUNG HSIEH ${ }^{1,7}$, PO-LIN KUO ${ }^{2}$, MING-FENG HOU ${ }^{2}$, JEN-YU HUNG $^{3}$, FANG-RONG CHANG $^{4}$, \\ YING-CHAN HSU $^{7}$, YA-FANG HUANG ${ }^{7}$, EING-MEI TSAI ${ }^{1,5,6}$ and YA-LING HSU ${ }^{1,6}$ \\ ${ }^{1}$ Graduate Institute of Medicine, ${ }^{2}$ Institute of Clinical Medicine, ${ }^{3}$ School of Medicine, College of Medicine, \\ ${ }^{4}$ Graduate Institute of Natural Products, ${ }^{5}$ Center for Research Resources and Development, \\ ${ }^{6}$ Research Center for Environmental Medicine, Kaohsiung Medical University; \\ ${ }^{7}$ Department of Chinese Medicine, Kaohsiung Medical University Hospital, Kaohsiung 807, Taiwan, R.O.C.
}

Received September 4, 2014; Accepted October 15, 2014

DOI: 10.3892/ijo.2014.2769

\begin{abstract}
The bone is the most common metastatic site of breast cancer. Bone metastasis causes pain, pathologic fractures, and severely reduces the quality of life. Breast cancer causes osteolytic bone metastasis, which is dependent on osteoclast-mediated bone resorption. While current treatments rely on palliative anti-resorptive agents, there is a need to develop a drug based on potential alternative therapies. This study is the first to determine that wedelolactone (WDL), a natural coumarin isolated from plants, can inhibit breast cancermediated osteoclastogenesis. Osteoclasts were generated from human $\mathrm{CD} 14^{+}$monocytes cultured with M-CSF/RANKL and WDL suppressed human osteoclast differentiation and activity in vitro in a dose-dependent manner. Moreover, WDL inhibited the upregulation of osteoclasts stimulated by MDA-MB-231 breast cancer cells. The activity of WDL on osteoclasts and breast cancer-mediated osteoclastogenesis was associated with the inhibition of $\mathrm{Akt} / \mathrm{mammalian}$ target of the rapamycin signaling pathway (mTOR). Blocking Akt and mTOR by specific inhibitors significantly decreased osteoclast differentiation and bone resorption. Furthermore, WDL regulated breast cancer-enhanced interaction of osteoblasts and osteoclasts by decreasing M-CSF expression in MDA-MB-231-stimulated osteoblasts. Thus, this study suggests that WDL may be a potential natural agent for preventing and treating bone destruction in patients with bone metastasis due to breast cancer.
\end{abstract}

\section{Introduction}

Breast cancer is the most common female cancer in the world and bone metastasis occurs in up to $70-80 \%$ of patients with

Correspondence to: Professor Eing-Mei Tsai or Dr Ya-Ling Hsu, Graduate Institute of Medicine, College of Medicine, Kaohsiung Medical University, Kaohsiung 807, Taiwan, R.O.C.

E-mail: tsaieing@yahoo.com

E-mail: hsuyl326@gmail.com

Key words: wedelolactone, osteoclastogenesis, breast cancer, bone metastasis advanced breast cancer $(1,2)$. Bone metastasis of breast cancer typically leads to osteolysis and results in bone destruction and skeletal-related events, including severe bone pain, pathologic fractures, spinal cord and nerve compression syndromes, and life-threatening hypercalcemia, which are a common cause of morbidity and mortality (3). Current treatments for bone metastasis have limited efficacy and are only palliative (4). Side-effects like renal toxicity and osteonecrosis of the jaw are considered clinically significant, potentially painful, and debilitating enough to decrease life quality of breast cancer patients $(2,5,6)$. Thus, it is important that therapeutic strategies are developed for the prevention and treatment of bone metastasis in breast cancer.

The spread of breast cancer to the bone begins with the initiation of bone demolition, the entry of malignant cells in the bone marrow, and subsequently, an increase in osteolysis (7). Bone metastasis is characterized by the complicated interaction between cancer cells and bone micro-environment (i.e., osteoclasts, osteoblasts and stroma cells) (7). Osteolytic bone metastasis is driven by the 'vicious cycle' of cancermediated upregulation of osteoclasts and bone stroma-induced cancer progression (8). Osteoclasts are large, multi-nucleated cells derived from hematopoietic precursor cells of the monocyte/macrophage lineage (9). Their differentiation is mediated by two cytokines, including macrophage colony stimulating factor (M-CSF) and receptor activator of nuclear factor $\kappa \mathrm{B}$ ligand (RANKL) (10). RANKL is crucial for osteoclast function by binding to its receptor RANK, while M-CSF maintains the proliferation and survival of osteoclasts, and upregulates RANK receptor expression in osteoclast precursor cells (11).

In addition to osteoclasts, breast cancer cells can also interact with osteoblasts to support osteoclast formation (12). Breast cancer cells increase the expressions of osteoclast differentiation factors, M-CSF and RANKL, and decrease osteoprotegerin (OPG) level in osteoblasts, leading to osteoclastogenesis (13-15). Therefore, molecular targeting of both cancer-mediated osteoclastogenesis or mending the imbalance of osteoblast-osteoclast interaction can reduce bone destruction (osteolytic bone metastasis).

Wedelolactone (7-methoxy-5,11,12-trihydroxy-coumestan, Fig. 1A) (Sigma-Aldrich, St. Louis, MO, USA) is a natural 
coumarin isolated from Wedelia chinensis and Eclipta prostrata, which are traditionally used for hair and skin health and for preventing liver damage due to alcohol overdose (16-19). Previous studies show that WDL has diverse pharmacologic effects such as anti-hepatotoxic, anti-androgenic, and antihuman immunodeficiency activities (19-21). Recent research has highlighted the antitumor effects of WDL. Combinations of active compounds in W. chinensis, including WDL, apigenin, and luteloin, have been found to suppress the growth of prostate cancer cells in vitro and in vivo $(20,22)$. Treatment with WDL inhibits the growth of pituitary adenoma cells and mammary carcinosarcomas in vitro $(23,24)$. Moreover, WDL can induce caspase-dependent apoptosis in prostate cancer cells via downregulation of PKCE (25).

This study demonstrates that WDL inhibits MDA-MB-231mediated osteoclastogenesis through the downregulation of the Akt/mTOR signaling pathway. Moreover, WDL downregulates M-CSF expression in MDA-CM-stimulated osteoblasts and inhibits osteoblast-mediated osteoclastogenesis.

\section{Materials and methods}

Cell culture. Human breast cancer MDA-MB-231 cells were purchased from the American Type Culture Collection (ATCC) (Manassas, VA, USA). MDA-MB-231 cells were cultured in standard modified essential medium ( $\alpha$-MEM) [ $\alpha$-MEM supplemented with $10 \%$ fetal calf serum (FCS), penicillin, and streptomycin]. Human primary osteoblasts were obtained from Lonza (Walkersville, MD, USA) and cultured in osteoblast growth medium.

For conditioned medium (CM) collection, the MDA-MB-231 cells were treated with vehicle $(0.1 \%$ DMSO) or various concentrations of WDL for $12 \mathrm{~h}$. After washing, fresh $\alpha$-MEM was added and cultured for another $24 \mathrm{~h}$. The supernatants were collected, filtered $(0.22 \mathrm{~mm})$, and defined as MDA-CM or WDL-MDA-CM. Osteoblasts were cultured with MDA-CM or WDL-MDA-CM (20\%) for another $24 \mathrm{~h}$ and the supernatants were then collected and filtered $(0.22 \mathrm{~mm})$. The collected supernatants were grouped as osteoblast-CM (OB-CM), MDA-OB-CM and WDL-MDA-OB-CM.

Osteoclast differentiation. Human peripheral blood, obtained from healthy adult volunteers, was collected in syringes containing $1000 \mathrm{U} / \mathrm{ml}$ of preservative-free heparin. The PBMCs were isolated by density centrifugation using Ficoll-Hypaque and were re-suspended in RPMI supplemented with $10 \%$ heat-inactivated fetal bovine serum. The PBMCs were then plated and incubated overnight at $37^{\circ} \mathrm{C} . \mathrm{CD} 14^{+}$monocytes were isolated using $\mathrm{CD}^{+} 4^{+} \mathrm{mAb}$-conjugated magnetic beads (MACS MicroBeads; Miltenyi Biotec), according to the manufacturer's protocol. Monocytes were grown in culture medium containing vehicle or $200 \mathrm{ng} / \mathrm{ml}$ human M-CSF and $100 \mathrm{ng} / \mathrm{ml}$ human RANKL for 14-21 days. The medium was replaced every five days. The Institutional Review Board of the Kaohsiung Medical University Chung-Ho Memorial Hospital approved the study protocol and all patients provided written informed consent in accordance with the Declaration of Helsinki.

Osteoclast formation was measured by quantifying cells positively stained by tartrate-resistant acid phosphatase (TRAP) (Sigma-Aldrich). Briefly, cells were fixed by form- aldehyde for $30 \mathrm{~min}$ and then stained with naphthol AS-BI phosphate and a tartrate solution for $1 \mathrm{~h}$ at $37^{\circ} \mathrm{C}$, followed by counter-staining with a hematoxylin solution. Osteoclasts were determined to be TRAP-positive staining multi-nuclear ( $>3$ nuclei) cells by light microscopy. The total number of TRAP-positive cells and number of nuclei per TRAP-positive cell in each well were counted.

Bone resorption assay. $\mathrm{CD} 14^{+}$monocytes were plated into a calcium phosphate apatite-coated 48-well plate bone resorption assay (Cosmo Bio Co., Ltd., Tokyo, Japan) in the same culture conditions as described above. After a 14-day culture, each well was washed with saline. A solution of 5\% sodium hypochlorite was left in the well for $5 \mathrm{~min}$ to detach the cells. The number of pits in each well was counted under a microscope.

Immunoblotting. Cells were lysed on ice for $15 \mathrm{~min}$ by M-PER lysis reagent (Thermo Fisher Scientific, Rockford, IL, USA). Cell lysate was centrifuged at $14,000 \mathrm{x}$ g for $15 \mathrm{~min}$ and the supernatant fraction was collected for immunoblotting. Equivalent amounts of protein were resolved by SDS-PAGE (6-8\%) and transferred to polyvinylidene difluoride membranes. After blocking for $1 \mathrm{~h}$ in $5 \%$ non-fat dry milk in TBS, the membrane was incubated with the desired primary Ab for 1-16 h. The membrane was then treated with appropriate peroxidase-conjugated secondary $\mathrm{Ab}$ and the immuno-reactive proteins were detected using an ECL kit (Millipore), according to the manufacturer's instructions.

Enzyme-linked immuno-sorbent assay (ELISA). The levels of M-CSF were assessed by M-CSF ELISA kit (R\&D Systems, Minneapolis, MN, USA). The RANKL and OPG levels of osteoblast were quantified using the DuoSet ELISA kits (R\&D Systems).

Statistical analysis. Data were expressed as means \pm SD. Statistical comparisons were made using analysis of variance. Significant differences $(\mathrm{p}<0.05)$ between two test groups were analyzed by Student's t-test.

\section{Results}

WDL exhibits direct inhibitory effect on osteoclast differentiation and bone resorption activity. To investigate if novel agents can inhibit osteoclasts, the effects of various phytoestrogens, including calycosin, formononetin, neobavaisoflavone, ononin, pinoresinol, and wedelolactone (WDL), on osteoclast differentiation were assessed. The addition of RANKL and M-CSF caused the formation of numerous TRAP-positive multi-nucleated osteoclasts (Fig. 1B). The differentiation of osteoclasts was significantly inhibited by WDL but other agents failed to affect osteoclastogenesis. Treatment of WDL decreased osteoclastogenesis in a dose-dependent manner (Fig. 1C). Moreover, WDL treatment substantially reduced osteoclastic bone resorption in a dose-dependent manner (Fig. 1D). These findings suggested that WDL inhibited osteoclast differentiation and bone resorption activity in vitro.

WDL inhibited breast cancer-mediated osteoclastogenesis. The effect of WDL on breast cancer-mediated osteoclasto- 
A<smiles>COc1cc(O)c2c(c1)oc(=O)c1c3cc(O)c(O)cc3oc21</smiles>
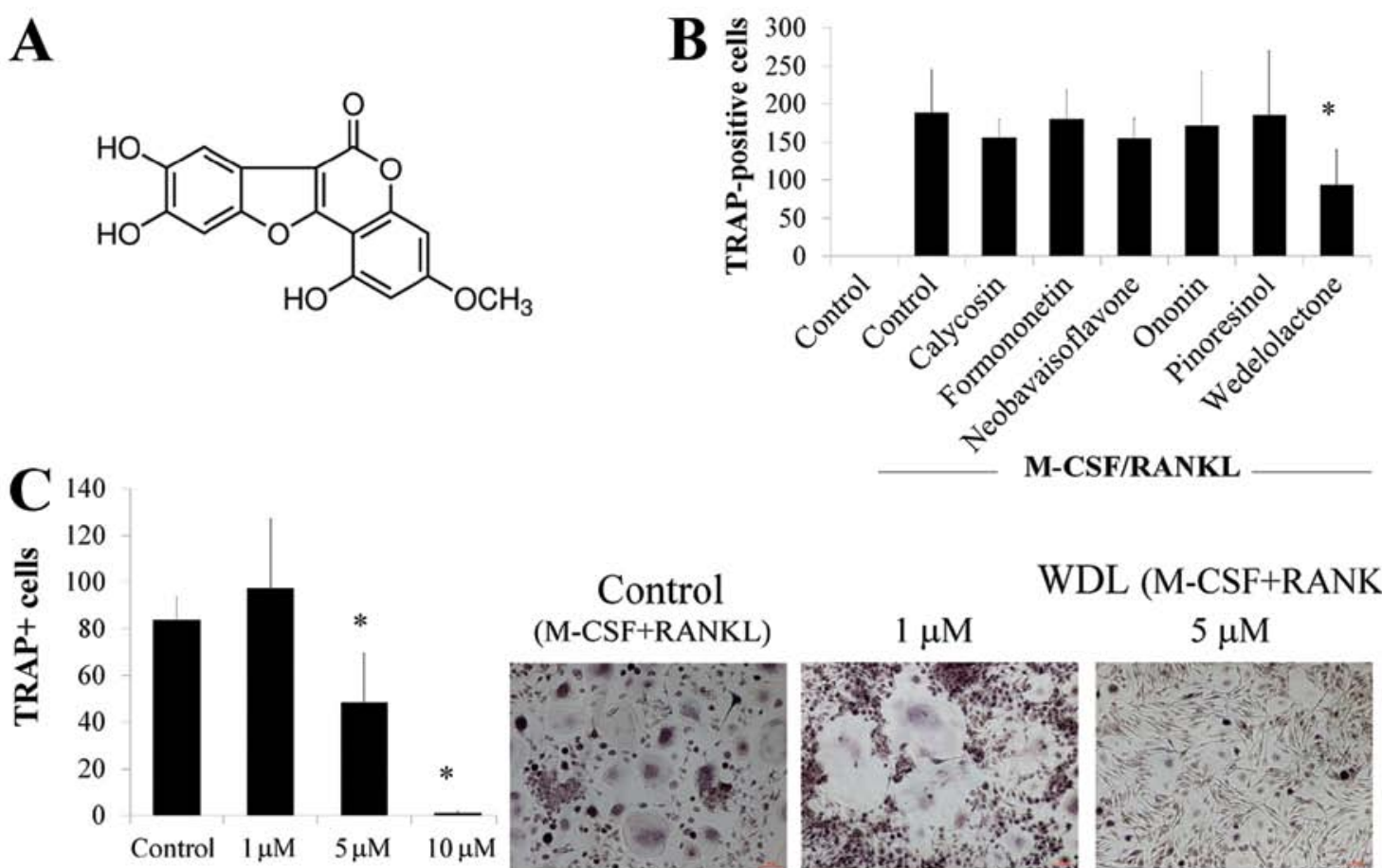

WDL concentration

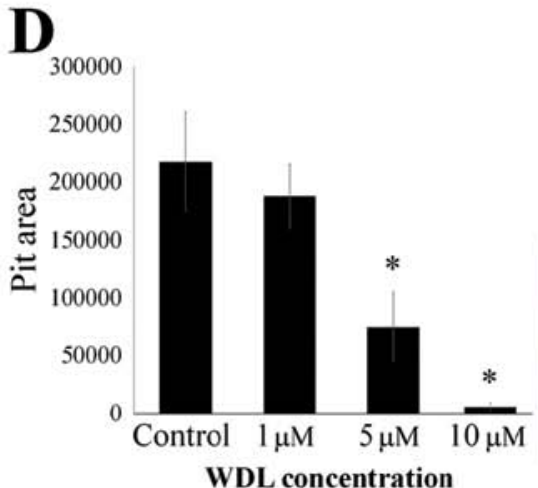

Control (M-CSF+RANKL)

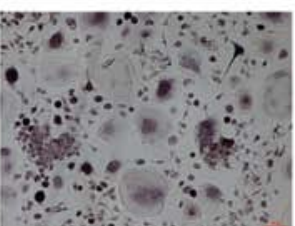

WDL (M-CSF+RANKL)

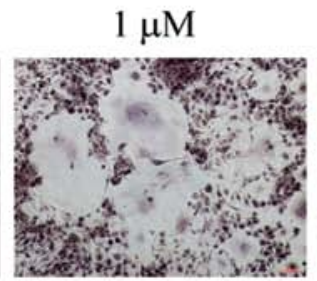

$5 \mu \mathrm{M}$

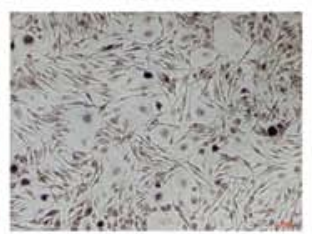

$10 \mu \mathrm{M}$

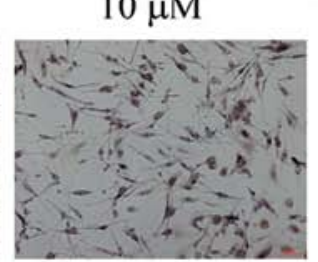

\section{D}

Figure 1. WDL decreased osteoclastogenesis. (A) The chemical structure of WDL. (B) The effect of various phytoestrogens in osteoclastogenesis. WDL decreased (C) osteoclast differentiation and (D) bone resorption. Each value is the mean \pm SD of three independent experiments. The pit area was determined by AlphaEase FC software. *p<0.05, or significant difference with controls, as analyzed by Student's t-test.

genesis was evaluated (Fig. 2A). MDA-MB-231-CM markedly increased osteoclastogenesis (TRAP-positive multi-nucleated osteoclasts) (Fig. 2B). This stimulatory effect of breast cancer MDA-MB-231 cells was significantly decreased by WDL. MDA-MB-231-CM also enhanced the bone resorption activity of osteoclasts and this effect of breast cancer was also reduced by WDL (Fig. 2C). These results indicated that WDL inhibited MDA-MB-231 cell-mediated osteoclastogenesis and osteoclast activity.

WDL decreased osteoclastogenesis by inhibiting Akt/mTOR. The molecular mechanism of WDL on osteoclastogenesis was investigated. The addition of M-CSF/RANKL increased the phosphorylation of Akt, mTOR, ERK1/2 and p38. The targets of mTOR, p70S6K, and 4EBP4 were also activated by M-CSF/RANKL, showing that Akt/mTOR signaling cascade was triggered in osteoclastogenesis. The activation of osteoclast inducers (M-CSF and RANKL) on Akt/mTOR signaling was inhibited by WDL (Fig. 3A).
The role of Akt/mTOR on osteoclastogenesis and bone resorption was further supported by using both Akt (Akt inhibitor IV) and mTOR inhibitors (rapamycin), which decreased osteoclastogenesis and its activity (Fig. 3B and C). In addition, WDL not only decreased M-CSF/RANKL-mediated $\mathrm{Akt} / \mathrm{mTOR}$ activation, but also reduced the reinforcing effect of breast cancer on this signaling pathway (Fig. 3D). These results revealed that WDL inhibited the Akt/mTOR signaling pathway, which was critical in osteoclastogenesis induced by M-CSF/RANKL and MDA-MB-231 cells.

WDL decreased the stimulatory effect of breast cancer on $M-C S F$ expression in osteoblasts. Osteoblasts are known to play a critical role on the deterioration of bone metastasis by increasing M-CSF and RANKL, and decreasing OPG expression (13-15). As such, the effect of WDL on the secretion of M-CSF, RANKL, and OPG in osteoblasts after stimulation of breast cancer was assessed. MDA-MB-231-CM markedly increased the expressions of M-CSF and RANKL in osteo- 


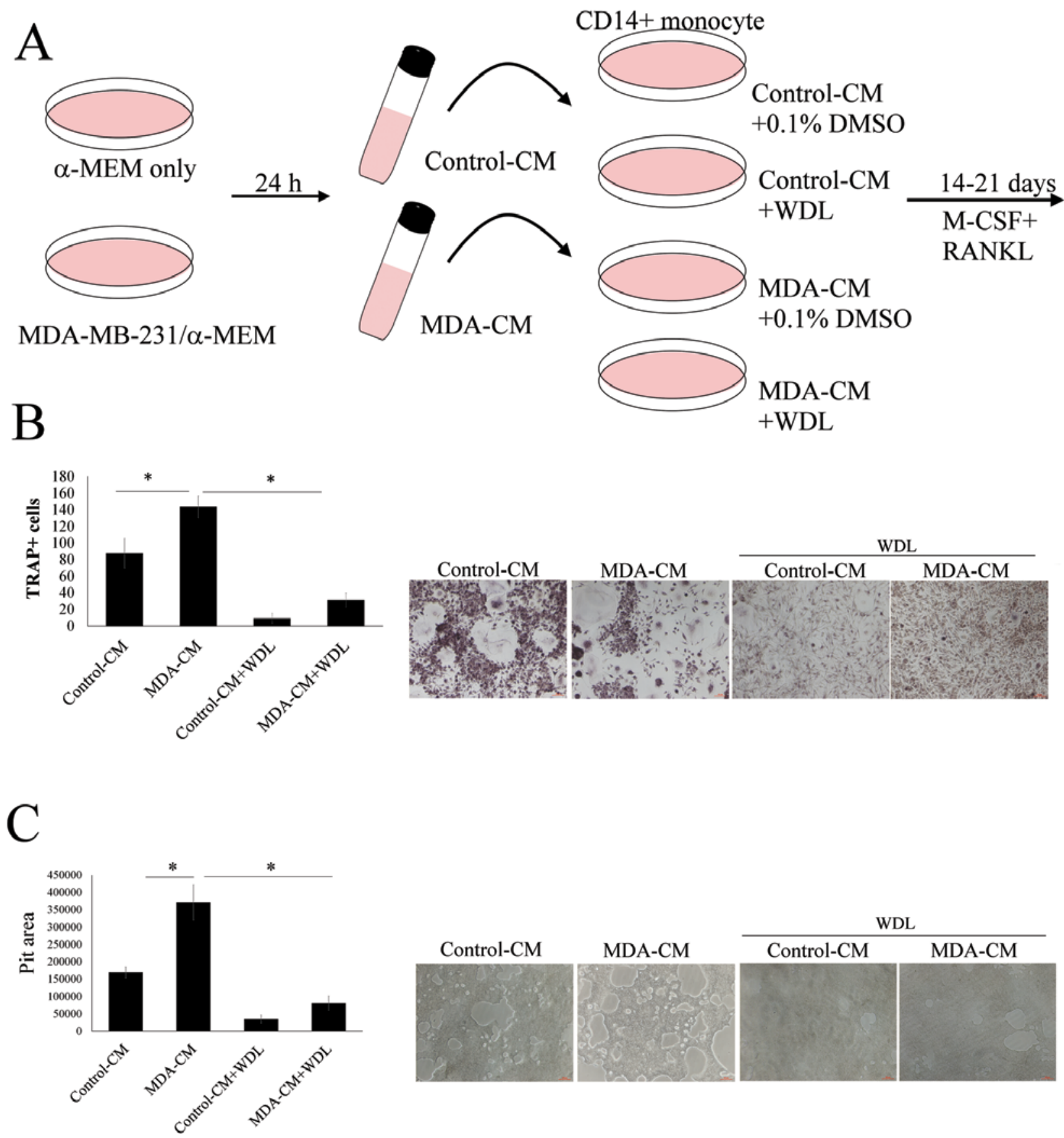

Figure 2. WDL inhibited breast cancer-mediated osteoclastogenesis. (A) Flow chart of production of control-CM and MDA-CM. WDL decreased MDA-MB-231mediated (B) osteoclast differentiation and $(C)$ bone resorption. Each value is the mean \pm SD of three independent experiments. The pit area was determined by AlphaEase FC software. "p $<0.05$, or significant difference with controls or two tested groups, as analyzed by Student's t-test.

blasts (Fig. 4A and B). Furthermore, MDA-MB-231-CM also decreased OPG expression in osteoblasts (Fig. 4C). WDL decreased the stimulatory effect of breast cancer on the production of M-CSF, although it did not affect the expressions of RANKL and OPG in osteoblasts (Fig. 4).

WDL reduces the enhancement effect of breast cancer on osteoblast-mediated osteoclastogenesis. The activity of WDL on breast cancer-stimulated interaction of osteoblasts and osteoclasts was further investigated (Fig. 5A). Breast cancer MDA-MB-231 stimulated osteoblast-induced osteoclastogenesis, compared to results of the osteoclastogenesis cultured in unstimulated osteoblast supernatants (Fig. 5B). The stimulatory effect of MDA-MB-231 cells was inhibited by WDL treatment. Similarly, breast cancer MDA-MB-231 cells increased the promotion of osteoblasts on osteoclast bone resorption and this effect was prevented by WDL (Fig. 5C). These findings suggest that WDL inhibited the deterioration of osteoblasts on breast cancer-mediated osteolytic bone metastasis.

\section{Discussion}

Bone metastasis is a devastating development in patients with breast cancer because once this occurs, morbidity and 


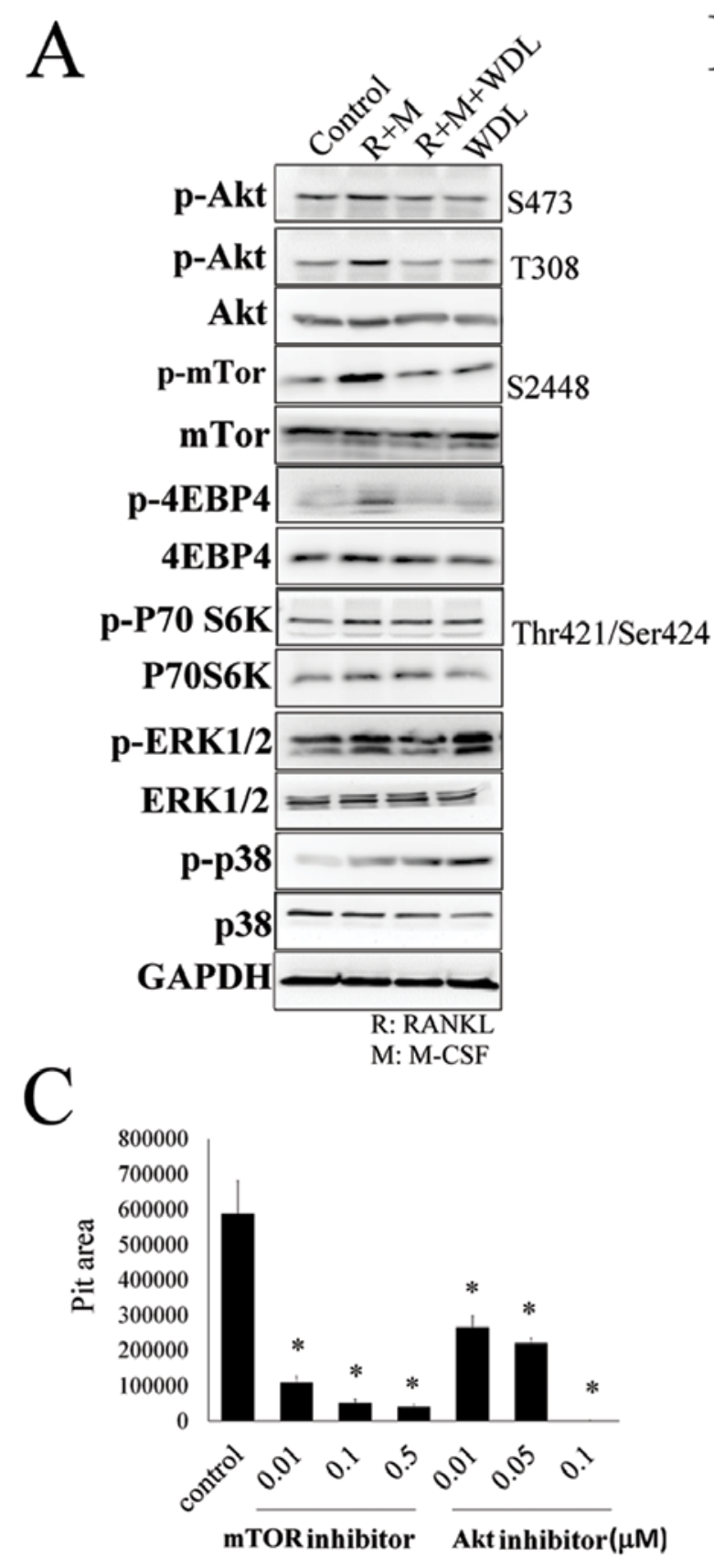

B
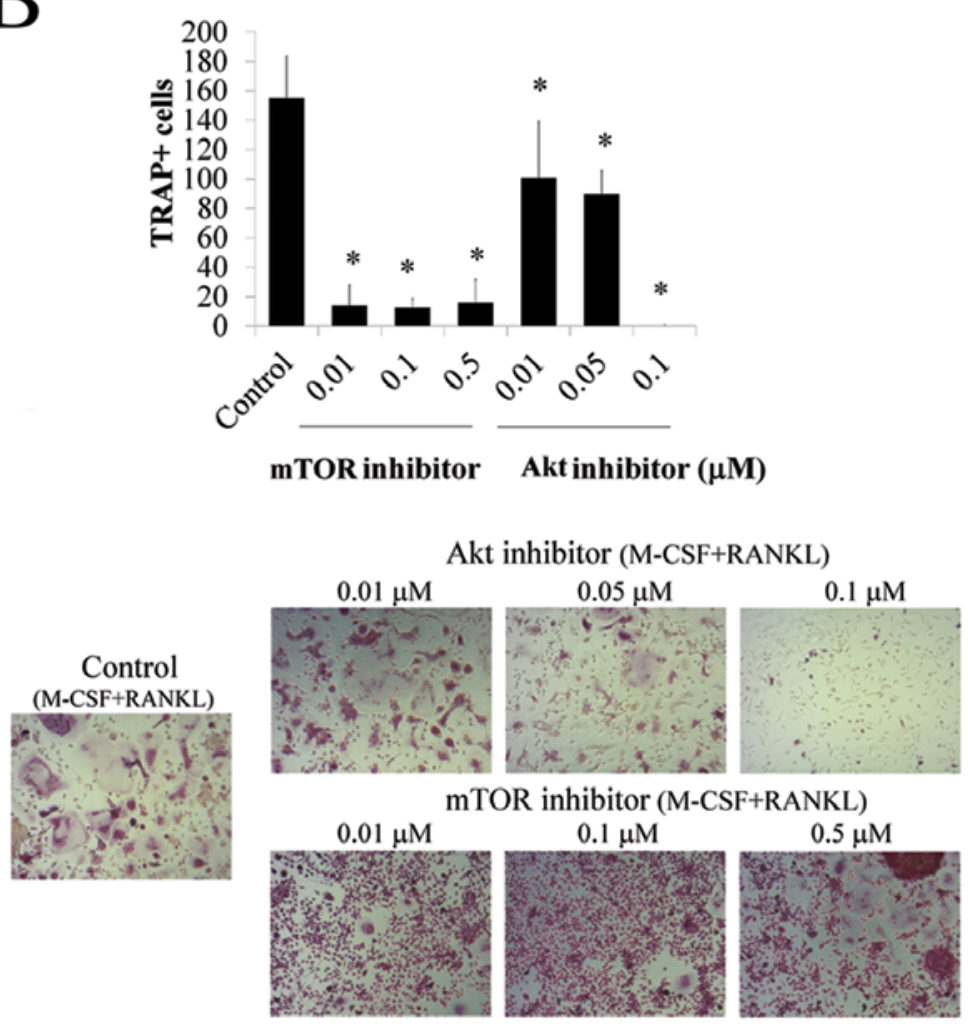

$\mathrm{D}$
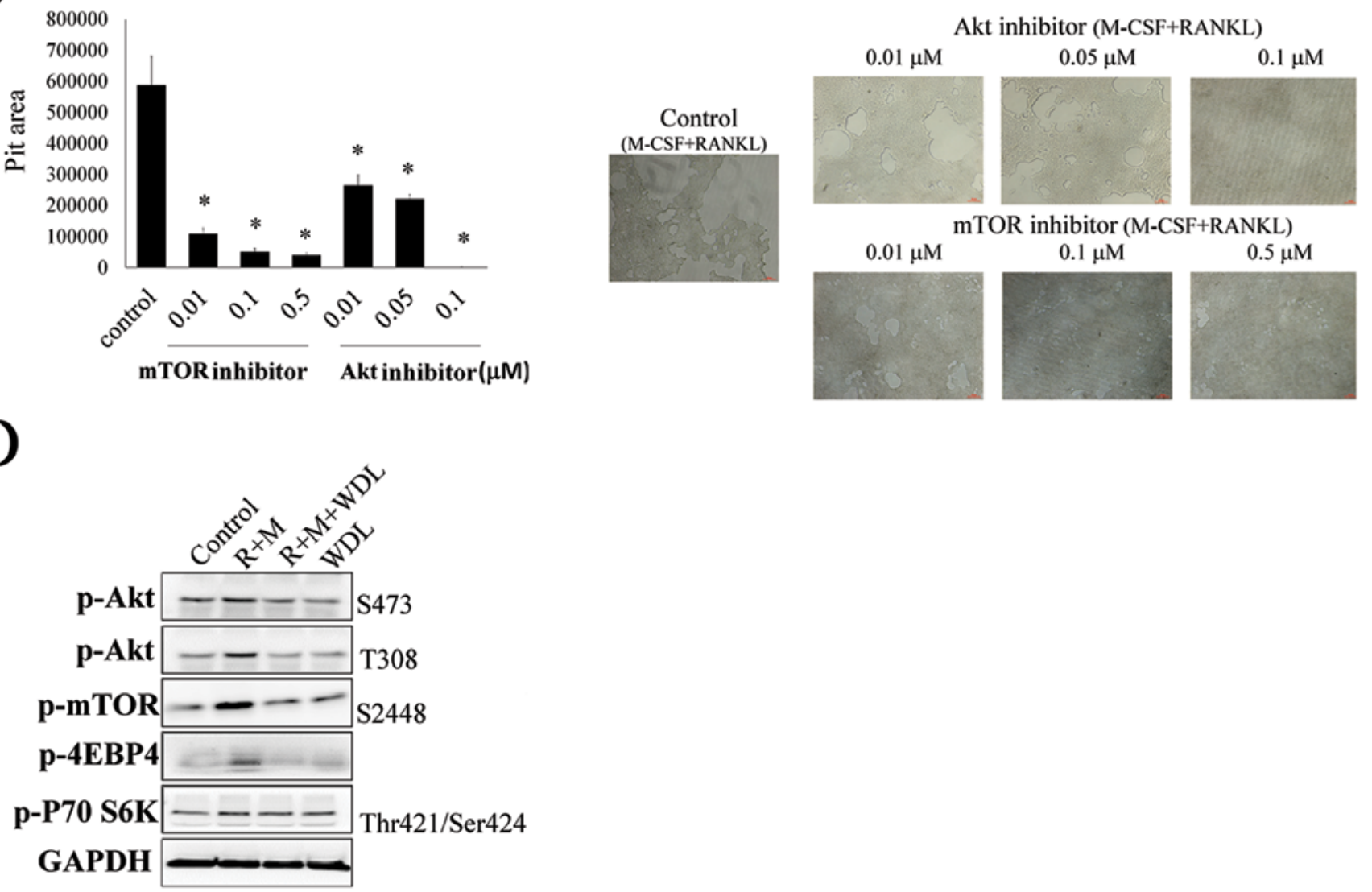

Figure 3. WDL decreases osteoclastogenesis by inhibiting Akt/mTOR signaling. (A) WDL reduced M-CSF/RANKL-mediated Akt signaling activation. Akt (Akt inhibitor IV) and mTOR (rapamycin) inhibitors decreased (B) osteoclast differentiation and (C) bone resorption. (D) WDL inhibited MDA-MB-231mediated Akt/mTOR activation. Each value is the mean $\pm \mathrm{SD}$ of three independent experiments. The pit area was determined by AlphaEase FC software. ${ }^{*} \mathrm{p}<0.05$, or significant difference with controls, as analyzed by Student's t-test. 


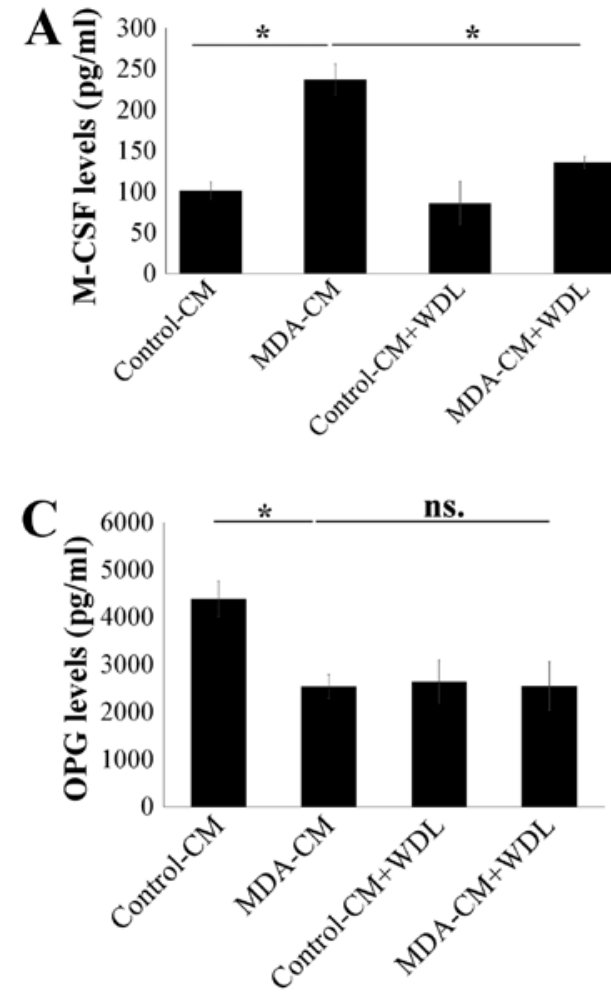

mortality rates are markedly increased $(2,4)$. Drugs that target osteoclastogenesis, such as bisphosphonates or anti-RANKL antibody (denosumab), decrease the incidence of skeletal complications and have been prescribed for the inhibition of cancer-mediated bone destruction (6). However, 30-50\% of patients on such therapies still develop new bone metastasis, skeletal complications, and disease progression, emphasizing the need for new therapies $(2,4,6)$. The present study is the first to demonstrate that WDL can inhibit breast cancer-induced osteolytic bone metastasis by decreasing the stimulatory effect of breast cancer on osteoclasts and breast cancer-mediated interaction of osteoblasts and osteoclasts.

Evaluation of osteoclast differentiation is characterized in breast cancer bone metastasis $(4,12)$. An increase in osteoclastic bone resorption leads to the release of bone-derived growth factors that promote the growth and progression of breast cancer cells $(4,12)$. Inhibiting osteoclast function provides a potential approach to preventing bone metastasis. Isolated from Eclipta prostrata and Wedelia chinensis, WDL belongs to the coumarin category of phytoestrogens. This is the first study that investigated and determined that WDL exhibits a direct inhibitory effect on osteoclast differentiation and bone resorption activity.

In addition, WDL also inhibits breast cancer MDA-MB-231mediated osteoclastogenesis and bone degradation. This finding suggests that WDL possesses therapeutic potential in the treatment of breast cancer bone metastasis. The impact of metastasized cancer cells on the bone interrupts the interaction between the activities of osteoclasts and osteoblasts (26). Osteoblasts perform vital roles in the regulation of skeleton physiology because they not only act as precursors of osteocytes but are also dual regulators of osteoclast differentiation $(27,28)$. Osteoblasts stimulate osteoclast formation from osteo-

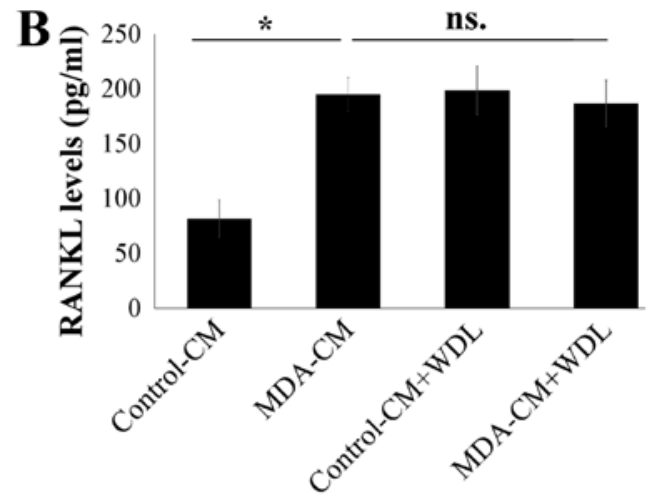

Figure 4. WDL decreases the upregulation of M-CSF in osteoblasts after breast cancer stimulation. WDL reduced the stimulatory effect of MDA-MB-231 on (A) M-CSF, but not on (B) RANKL and (C) OPG in osteoblasts. Each value is the mean \pm SD of three independent experiments. ${ }^{*} \mathrm{p}<0.05$, or significant difference with controls or two tested groups, as analyzed by Student's t-test.

clast precursors by producing M-CSF and RANKL (29). In contrast, osteoblasts inhibit osteoclastogenesis via secreting OPG, a decoy receptor for RANKL to block the binding of RANKL to its receptors on pre-osteoclasts, RANK (13).

Cancer can alter the expressions of M-CSF, RANKL, and OPG in osteoblasts, facilitating cancer-associated osteolytic bone metastasis $(14,30)$. In this study, breast cancer MDA-MB-231 cells increased the expressions of M-CSF and RANKL but decreased OPG expression, thereby increasing osteoclastogenesis and the bone resorption activity. This upregulation of M-CSF by MDA-MB-231 is prevented by WDL, resulting in a decrease in breast cancer-associated osteocleogenesis and bone resorption. This study revealed that WDL can mend the imbalanced interaction of osteoblasts and osteoclasts in breast cancer-mediated skeletal microenvironment.

Akt is a critical mediator of cell proliferation, survival and differentiation in a variety of cell types (31). Akt has been identified as being involved in the regulation of osteoclast survival and differentiation, whereas its deficiency in osteoclasts results in impaired bone resorption $(32,33)$. mTOR, a downstream molecule of Akt, has also been shown to be involved in the regulation of osteoclast survival (34). Inhibition of the mTOR pathway by rapamycin, an mTOR inhibitor, decreases the number of TRAP-positive multi-nucleated osteoclasts in the chondro-osseous junction in rats (35).

In this study, osteoclast differentiation induced by M-CSF/RANKL increased the activation of Akt and mTOR signaling cascade. Inhibitors of both Akt and mTOR markedly suppressed osteoclast differentiation and bone resorption activity, suggesting that the Akt/mTOR pathway plays a crucial role in osteoclastogenesis and osteoclast functions. Moreover, breast cancer MDA-MB-231 cells augment the activation of 

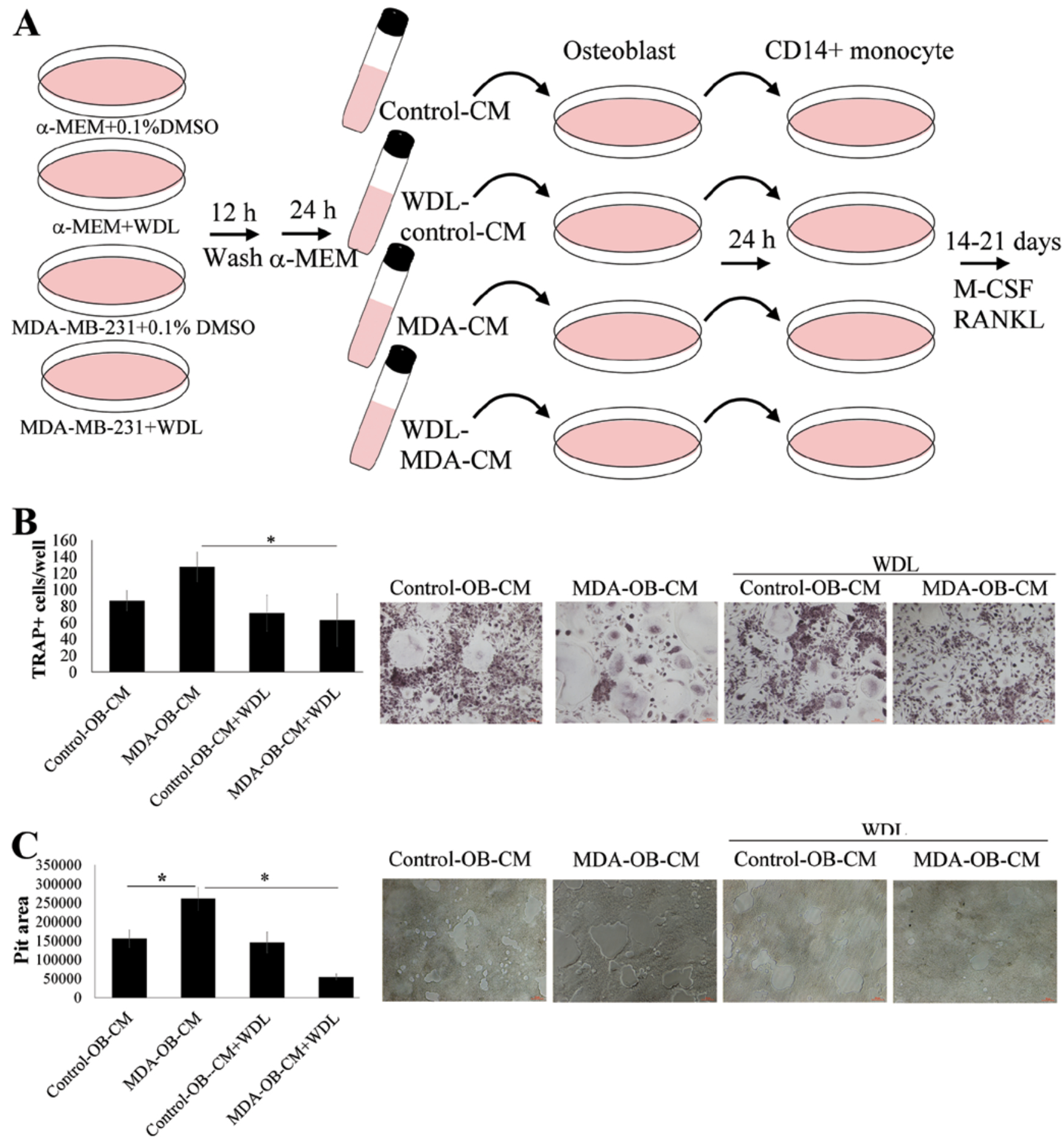

Figure 5. WDL decreased the stimulatory effect of breast cancer on osteoblast-mediated osteoclastogenesis. (A) Flow chart of production of control-CM, WDLcontrol-CM, MDA-CM, WDL-MDA-CM or various osteoblast conditioned medium. WDL reduced (B) osteoclast differentiation and (C) bone resorption activity induced by breast cancer-stimulated osteoblasts. Each value is the mean \pm SD of three independent experiments. The pit area was determined by AlphaEase FC software. ${ }^{*} \mathrm{p}<0.05$, or significant difference with controls, as analyzed by Student's t-test.

Akt/mTOR signaling, resulting in enhanced osteoclastogenesis. WDL not only blocks M-CSF/RANKL-induced Akt/ mTOR activation, but also prevents the reinforcing effect of breast cancer on this signaling pathway. These data suggest that WDL is an Akt/mTOR inhibitor, targeting both the induction of osteoclast differentiation and the cancer inducible activation of $\mathrm{Akt} / \mathrm{mTOR}$.

In conclusion, WDL has protective potential against breast cancer-induced bone destruction by directly decreasing cancer cell mediated osteoclast differentiation and bone resorption and by restoring the balance of osteoblast-osteoclast interaction. Taken together, WDL possesses potential and dual ameliorating effects on breast cancer-associated osteolytic bone metastasis.

\section{Acknowledgements}

This study was supported by grants from the National Science Council of Taiwan (NSC 102-2628-B-037-002-MY3; NSC 
102-2632-B-037-001-MY3; NSC 102-2314-B-037-035-MY3), the Excellence for Cancer Research Center Grant, the Ministry of Health and Welfare, Executive Yuan, Taipei, Taiwan (MOHW 103-TD-B-111-05), and the Kaohsiung Medical University Hospital (KMUH102-2M61). The authors thank the Center for Research Resources and Development of Kaohsiung Medical University for their support with the instrumentation.

\section{References}

1. Akhtari M, Mansuri J, Newman KA, Guise TM and Seth P: Biology of breast cancer bone metastasis. Cancer Biol Ther 7: 3-9, 2008.

2. Rordorf T, Hassan AA, Azim H, et al: Bone health in breast cancer patients: a comprehensive statement by CECOG/SAKK intergroup. Breast 23: 511-525, 2014

3. Rove KO and Crawford ED: Metastatic cancer in solid tumors and clinical outcome: skeletal-related events. Oncology 23: 21-27, 2009.

4. Weilbaecher KN, Guise TA and McCauley LK: Cancer to bone: a fatal attraction. Nat Rev Cancer 11: 411-425, 2011.

5. Gupta S, Gupta H, Mandhyan D and Srivastava S: Bisphophonates related osteonecrosis of the jaw. Natl J Maxillofac Surg 4: 151-158, 2013.

6. Verron E, Schmid-Antomarchi H, Pascal-Mousselard H, Schmid-Alliana A, Scimeca JC and Bouler JM: Therapeutic strategies for treating osteolytic bone metastases. Drug Discov Today 19: 1419-1426, 2014.

7. Roodman GD: Mechanisms of bone metastasis. N Engl J Med 350: 1655-1664, 2004.

8. Mundy GR: Metastasis to bone: causes, consequences and therapeutic opportunities. Nat Rev Cancer 2: 584-593, 2002.

9. Rodan GA and Martin TJ: Therapeutic approaches to bone diseases. Science 289: 1508-1514, 2000.

10. Suda T, Takahashi N, Udagawa N, Jimi E, Gillespie MT and Martin TJ: Modulation of osteoclast differentiation and function by the new members of the tumor necrosis factor receptor and ligand families. Endocr Rev 20: 345-357, 1999.

11. Lee $\mathrm{ZH}$ and Kim HH: Signal transduction by receptor activator of nuclear factor kappa B in osteoclasts. Biochem Biophys Res Commun 305: 211-214, 2003.

12. Chen YC, Sosnoski DM and Mastro AM: Breast cancer metastasis to the bone: mechanisms of bone loss. Breast Cancer Res 12: $215,2010$.

13. Mountzios G, Dimopoulos MA, Bamias A, et al: Abnormal bone remodeling process is due to an imbalance in the receptor activator of nuclear factor-kappaB ligand (RANKL)/osteoprotegerin (OPG) axis in patients with solid tumors metastatic to the skeleton. Acta Oncol 46: 221-229, 2007.

14. McGrath EE: OPG/RANKL/RANK pathway as a therapeutic target in cancer. J Thorac Oncol 6: 1468-1473, 2011.

15. Azim HA, Kamal NS and Azim HA Jr: Bone metastasis in breast cancer: the story of RANK-ligand. J Egypt Nat Cancer Inst 24: 107-114, 2012.

16. Patel M, Kadakia V and Mishra S: Simultaneous estimation of andrographolide and wedelolactone in herbal formulations. Indian J Pharm Sci 70: 689, 2008.

17. Roy RK, Thakur M and Dixit VK: Hair growth promoting activity of Eclipta alba in male albino rats. Arch Dermatol Res 300: 357-364, 2008.
18. Singh B, Saxena AK, Chandan BK, Agarwal SG and Anand KK: In vivo hepatoprotective activity of active fraction from ethanolic extract of Eclipta alba leaves. Indian J Physiol Pharmacol 45: 435-441, 2001

19. Wagner H, Geyer B, Kiso Y, Hikino H and Rao GS: Coumestans as the main active principles of the liver drugs Eclipta alba and Wedelia calendulacea. Planta Med: 370-374, 1986.

20. Lin FM, Chen LR, Lin EH, et al: Compounds from Wedelia chinensis synergistically suppress androgen activity and growth in prostate cancer cells. Carcinogenesis 28: 2521-2529, 2007.

21. Tewtrakul S, Subhadhirasakul S, Cheenpracha S and Karalai C: HIV-1 protease and HIV-1 integrase inhibitory substances from Eclipta prostrata. Phytother Res 21: 1092-1095, 2007.

22. Tsai CH, Lin FM, Yang YC, et al: Herbal extract of Wedelia chinensis attenuates androgen receptor activity and orthotopic growth of prostate cancer in nude mice. Clin Cancer Res 15: 5435-5444, 2009.

23. Vender JR, Laird MD and Dhandapani KM: Inhibition of NFkappaB reduces cellular viability in GH3 pituitary adenoma cells. Neurosurgery 62: 1122-1128, 2008.

24. Idris AI, Libouban H, Nyangoga H, Landao-Bassonga E, Chappard D and Ralston SH: Pharmacologic inhibitors of IkappaB kinase suppress growth and migration of mammary carcinosarcoma cells in vitro and prevent osteolytic bone metastasis in vivo. Mol Cancer Ther 8: 2339-2347, 2009.

25. Sarveswaran S, Gautam SC and Ghosh J: Wedelolactone, a medicinal plant-derived coumestan, induces caspase-dependent apoptosis in prostate cancer cells via downregulation of $\mathrm{PKC \varepsilon}$ without inhibiting Akt. Int J Oncol 41: 2191-2199, 2012.

26. Sterling JA, Edwards JR, Martin TJ and Mundy GR: Advances in the biology of bone metastasis: how the skeleton affects tumor behavior. Bone 48: 6-15, 2011.

27. Furugaki K, Moriya Y, Iwai T, et al: Erlotinib inhibits osteolytic bone invasion of human non-small-cell lung cancer cell line NCI-H292. Clin Exp Metastasis 28: 649-659, 2011.

28. Hsu YL, Huang MS, Yang CJ, Hung JY, Wu LY and Kuo PL: Lung tumor-associated osteoblast-derived bone morphogenetic protein-2 increased epithelial-to-mesenchymal transition of cancer by Runx2/Snail signaling pathway. J Biol Chem 286: 37335-37346, 2011.

29. Takayanagi $\mathrm{H}$ : New immune connections in osteoclast formation. Ann NY Acad Sci 192: 117-123, 2010.

30. Yoneda T, Tanaka S and Hata K: Role of RANKL/RANK in primary and secondary breast cancer. World J Orthop 4: 178-185, 2013.

31. Skeen JE, Bhaskar PT, Chen CC, et al: Akt deficiency impairs normal cell proliferation and suppresses oncogenesis in a p53-independent and mTORC1-dependent manner. Cancer Cell 10: 269-280, 2006.

32. Moon JB, Kim JH, Kim K, et al: Akt induces osteoclast differentiation through regulating the GSK3 $\beta /$ NFATc1 signaling cascade. J Immunol 188: 163-169, 2012.

33. Cao H, Zhu K, Qiu L, et al: Critical role of AKT protein in myeloma-induced osteoclast formation and osteolysis. J Biol Chem 288: 30399-30410, 2013.

34. Sugatani T and Hruska KA: Akt1/Akt2 and mammalian target of rapamycin/Bim play critical roles in osteoclast differentiation and survival, respectively, whereas Akt is dispensable for cell survival in isolated osteoclast precursors. J Biol Chem 280: 3583-3589, 2005.

35. Sanchez CP and He YZ: Bone growth during rapamycin therapy in young rats. BMC Pediatr 9: 3, 2009. 